\title{
Brucellosis: An Elusive Backyard Agent
}

\author{
Zain Rizvi ${ }^{1}$, Tahir Iqbal ${ }^{1}$, Haider Bokhary ${ }^{2}$, Shiza Chaudhry ${ }^{2}$ \\ 1. Internal Medicine, Shifa International Hospital, Islamabad, PAK 2. Medicine, Shifa International Hospital, \\ Islamabad, PAK
}

Corresponding author: Zain Rizvi, zainrizvi@live.ca

\begin{abstract}
Brucellosis is a form of zoonotic infection caused by various Brucella organisms. It most commonly presents as a case of pyrexia of unknown origin, alongside symptoms such as night sweats, malaise, arthralgias, and myalgias. This report describes the case of a man who presented with pyrexia of unknown origin for one month; he was diagnosed to be a case of brucellosis after enteric fever was ruled out. Investigations were ordered as it was a differential diagnosis with high clinical suspicion due to the presenting complaint and potential exposure of tainted consumable products. The systemic disease was determined to be brucellosis following blood results demonstrating positive antibody titers, and the suspicion of exposure due to widespread inadequacies in sterilization of food products.
\end{abstract}

Categories: Family/General Practice, Internal Medicine, Infectious Disease

Keywords: brucella, brucellosis, infectious disease, medicine, internal medicine, family medicine, contagious, zoonotic, zoonosis, pasteurization

\section{Introduction}

Brucellosis, also known as Mediterranean fever, is a zoonotic infection caused by animals infected with Brucella organisms, which are aerobic intracellular coccobacilli. Humans are accidental hosts; it is transmitted to them by means of infected animal fluids or tissue, or by ingestion of animal food products that have not been sterilized or pasteurized [1]. It presents with insidious onset of fever, night sweats, malaise, and arthralgias. Uncommonly, it can cause weight loss, cough, and abdominal pain [2].

The burden of the disease remains high, approximately 500,000 cases are reported worldwide every year, the majority being in the Middle East, Asia, the Indian Subcontinent, and South America [3,4]. The most common cause of infection is due to ingestion of food products that are not sterilized or pasteurized properly [1]. Furthermore, brucellosis caused due to infection from wildlife is another possible mode of transmission to humans or domestic animals that may pass it on to their handlers, which is a form of occupational risk associated with the disease [5]. Four organisms can cause infections in humans, each one being from a different infected species of animals. Brucella melitensis from sheep and goats, Brucella abortus from cattle, Brucella suis from swine, and Brucella canis isolated from dogs. From the listed species, the most virulent infection is due to sheep, goats, and swine [6].

Received 05/05/2020

Review began 05/11/2020 Review ended 05/11/2020 Published 05/16/2020

\section{() Copyright 2020}

Rizvi et al. This is an open access article distributed under the terms of the Creative Commons Attribution License CC-BY 4.0., which permits unrestricted use, distribution, and reproduction in any medium, provided the original author and source are credited.
Brucellosis typically presents with a fever in around $80 \%$ of patients; this leads to the diagnosis of fever of unknown origin in some areas where it is rare [7]. Secondly, chills are very common in these patients. Constitutional symptoms include weight loss, malaise, fatigue, and arthralgias, which are present in around $55 \%$ of presenting cases [8]. The nervous system is rarely involved but can lead to neuropsychiatric symptoms, such as headache, weakness, and depression. Furthermore, gastrointestinal symptoms typically range from dyspepsia to abdominal pain caused by hepatic abscesses $[1,8]$. Rarely there can be cough and dyspnea, these are typically due to an underlying empyema [9]. Lastly, in its chronic form which lasts for longer than one year, there is an afebrile pattern, alongside history of myalgia, fatigue and arthralgias. This frequently leads to a differential diagnosis of chronic fatigue syndrome $[8,10]$.

Hence, as brucellosis can mimic numerous diseases due to its varied symptoms, it is important to look for clues such as a person's occupation, the area that they live in, and know about the endemicity of Brucella in that region. Due to the organism's predominance in the region of the presenting patient, it was considered as a differential diagnosis of this patient's condition.

We illustrate a case of an old man who presented with a step ladder pattern fever, myalgias, dry cough, fatigue, and reduced appetite. Investigations showed a positive blood test for brucella immunoglobulin $\mathrm{M}$ (IgM) antibodies, alongside findings on x-ray and computed tomography (CT) scan.

\section{Case Presentation}

A 58-year-old retired man of Pakistani origin, known smoker, presented to our outpatient clinic with fever for the past one month. It was associated with painful body aches, dry cough, reduced appetite, and fatigue. The fever had been present for every day, and it was of a step ladder pattern, with a rise during the evening 
time. It achieved a maximum of $102^{\circ} \mathrm{F}$ in the evening and it reduced to approximately $99^{\circ} \mathrm{F}$ during the early morning with associated chills and rigors. The patient did not report any other symptoms. There was no significant past medical history. He was prescribed oral amoxicillin by a local doctor previously for his symptoms with no improvement noted.

On physical examination, an ill looking man with a body temperature of $101^{\circ} \mathrm{F}$, a pulse rate of $76 / \mathrm{min}$, a blood pressure of $120 / 70 \mathrm{mmHg}$, and a respiratory rate of 18 breaths/min. On inspection, there was no apparent rash, marks, or swellings present. On palpation, no tenderness was present. Respiratory and abdominal system examinations were normal and had no positive findings. There was no lymphadenopathy or hepatosplenomegaly.

Investigations revealed normal hemoglobin $(14.7 \mathrm{~g} / \mathrm{dL})$ and platelet count $\left(269 \times 10^{3} / \mathrm{mm}^{3}\right)$. However, white blood cell (WBC) counts were raised $\left(10,800 / \mathrm{mm}^{3}\right)$ and differential counts were skewed (neutrophils $66 \%$, lymphocytes $20 \%$, monocytes $9 \%$, and eosinophils 5\%). Further blood biochemistry showed normal blood sugar fasting (108 mg/dL) and thyroid function tests; he had low levels of folic acid $(2.3 \mathrm{ng} / \mathrm{mL})$ and 25 hydroxy vitamin $\mathrm{D}(11.39 \mathrm{ng} / \mathrm{mL})$. Liver function tests revealed mildly raised serum glutamic pyruvic transaminase (SGPT) (43 IU/L) and increased levels of gamma-glutamyl transferase (GGT) (94 U/L). Creactive protein (CRP) and erythrocyte sedimentation rate (ESR) were measured and were increased (71.62 $\mathrm{mg} / \mathrm{L}$ and $49 \mathrm{~mm} /$ first hour). Malarial infection was excluded out by means of a negative blood smear test. Sputum smear for acid fast bacilli revealed no growth. However, blood test for brucella IgM antibodies was positive (1.31). Initial chest radiograph did not show any anomaly.

Due to the prolonged pyrexia of unknown origin initially, a preliminary diagnosis of enteric fever was made. He was admitted to the hospital in-patient department under the care of a specialist in internal medicine. The initial diagnosis was suspected to be enteric fever, based on the endemicity in the area, and accordingly he was started on azithromycin and ceftriaxone therapy. Blood tests done later during the admission revealed the presence of brucella antibodies, leading to the definitive diagnosis of brucellosis and change in treatment plan.

Furthermore, imaging studies three days after admission consisted of a chest radiograph, which showed mildly prominent subcentimeteric mediastinal hilar lymph nodes and emphysematous changes in lungs (Figure 1). CT of the chest was ordered, and it showed multiple subcentemetric mediastinal hilar lymphadenopathy. The diagnosis of brucellosis was made due to the presence of brucella antibodies in the blood report. Additionally, this seemed like an appropriate diagnosis due to Brucella being silently prevalent in the rural areas of Pakistan. Human brucellosis is known to cause a wide array of symptoms, of which some were present in the presenting case. Following confirmation of brucellosis, he was treated with doxycycline (100 mg twice daily) and rifampicin (600 mg once daily). After treatment was completed, the patient was discharged with a three-week follow-up to the medical clinic. 


\section{Cureus}

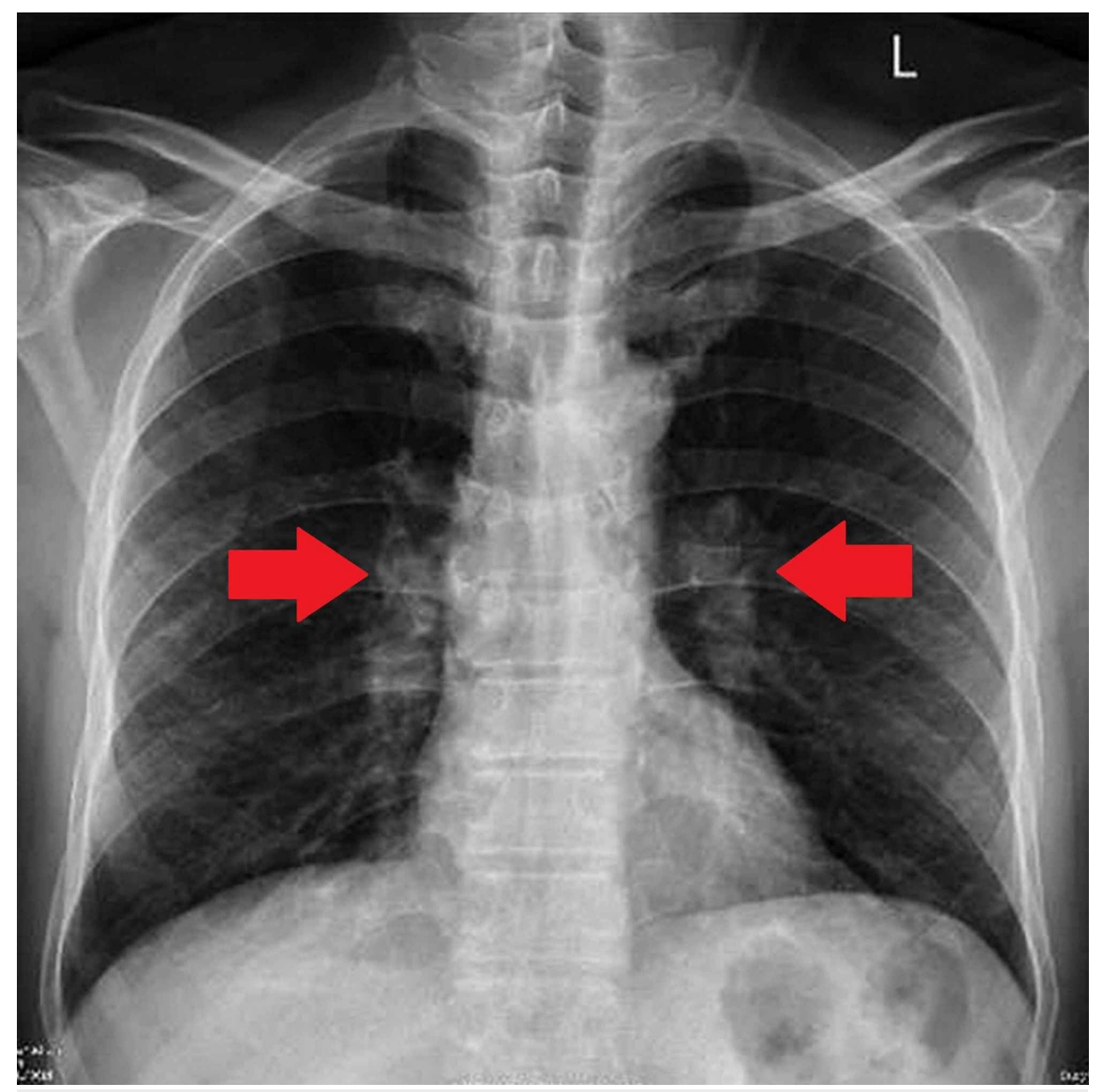

FIGURE 1: Chest x-ray before treatment with doxycycline with presence of bilateral hilar lymphadenopathy (red arrows).

\section{Discussion}

Brucellosis is a condition that puts enormous burdens in the countries where it is endemic. The fact that it is endemic to countries mostly lacking adequate resources and infrastructure to diagnose all cases leads to a high possibility that most cases of brucellosis remain undetected under the guise of pyrexia of unknown origin. This is likely one of the reasons the World Health Organization (WHO) has categorized it as a "neglected zoonotic disease" [11]. With early detection and the right treatment, the prognosis of brucellosis is generally excellent. However, if left untreated, it can lead to several complications ranging from neurobrucellosis to endocarditis, conditions which significantly increase the threat of morbidity and mortality of the disease [12]. Therefore, recognizing the signs and symptoms of brucellosis, and subsequently diagnosing and treating the patient are of paramount importance.

Fever is the most common symptom of an acute infection with Brucella, while in chronic cases the most common symptom is arthralgias [13]. The fever can develop either suddenly or insidiously and more commonly follows an undulant pattern. Several other symptoms can develop along with fever and arthralgias as was the case in the patients being reported, with other symptoms including cough, loss of appetite, and fatigue. Another rare symptom is testicular pain and swelling in patients with active brucellosis, with one study reporting an incidence of $10 \%$ [13]. There are several cases where enlargement of liver, spleen, and lymph nodes has been observed along with the fever, as a result of which it becomes important to rule out lymphomas as well [14]. This was also seen in our patient with the development of hilar lymphadenopathy on follow-up chest radiography.

Hematological parameters are frequently disturbed in brucellosis. While our patient's blood picture revealed leukocytosis $\left(10,800 / \mathrm{mm}^{3}\right)$ with a normal red cell and platelet count, the more common findings in brucellosis include leukopenia and relative lymphocytosis along with anemia, thrombocytopenia, and rarely pancytopenia [15]. Hypersplenism, which was absent in our patient, appears to play a fundamental role in producing these abnormalities of the peripheral blood [15]. Like in any other infection, elevation in inflammatory markers like ESR and CRP is expected to be seen. In certain studies, $38 \%$ to $87 \%$ and $34 \%$ to $81 \%$ of patients with brucellosis had elevated ESR and CRP, respectively [16]. As expected, our patient 
showed increased levels of both inflammatory markers. Similarly, a slight elevation in liver enzyme levels is a very common finding, as was seen with a slight increase in SGPT and GGT in the patient. These elevated levels may reflect the severity of hepatic involvement and correlate clinically with hepatomegaly.

Multiple organ systems can be affected due to brucellosis, resulting in a variety of clinical presentations. Our patient reported experiencing dry cough; subsequent chest radiography and CT showed subcentimeteric mediastinal hilar lymphadenopathy. Although pulmonary involvement is rare in brucellosis, the following radiological abnormalities have been reported: miliary mottling, parenchymal nodules, pleurisy, lung abscess, bronchiectasis, pneumonic consolidation, hilar or paratracheal lymphadenopathy, and pneumothorax [17].

The standard treatment of choice for brucellosis according to literature comprises a doxycyclineaminoglycoside combination as the first choice, followed by doxycycline-rifampin and doxycyclinecotrimoxazole as alternatives. For children younger than eight years of age, the antibiotic combination used is cotrimoxazole and rifampin [18]. Our patient received a combination of doxycycline and rifampicin, which led to recovery.

It is important to note that our patient was not affiliated with an occupation predisposed toward acquiring the infection. Therefore, it becomes vital to gather data regarding diet, travel and occupation from patients suspected of brucellosis. It has been observed that the most common risk factors for development of the infection are consumption of unpasteurized milk (48\%) and contact with livestock animals (59\%) [19]. Occupations predisposed toward such exposure most commonly include farmers, livestock breeders, and shepherds, but are also common among veterinarians and laboratory workers [20].

\section{Conclusions}

Brucellosis is a condition considered by the WHO to be "neglected zoonotic disease," which, although has good prognosis with the right treatment, can become much more complicated if left undetected and untreated. It is also rare enough a condition to be missed among the many causes of fever of unknown origin especially in areas with lower endemicity. What aided us in the diagnosis in our patient were the relatively typical presenting symptoms having lasted for one month, the infrequent pasteurization of milk in our country, and the access to adequate diagnostic facilities in our set up to detect a rise in WBC counts, ESR, CRP, and the eventual detection of brucella IgM antibodies, basic facilities which are still not readily accessible to many people of our country. This study highlights how it may be an important cause of fever even in patients naïve to the typical predisposing factors and in areas of the world not thought to be particularly endemic.

\section{Additional Information \\ Disclosures}

Human subjects: Consent was obtained by all participants in this study. Conflicts of interest: In compliance with the ICMJE uniform disclosure form, all authors declare the following: Payment/services info: All authors have declared that no financial support was received from any organization for the submitted work. Financial relationships: All authors have declared that they have no financial relationships at present or within the previous three years with any organizations that might have an interest in the submitted work. Other relationships: All authors have declared that there are no other relationships or activities that could appear to have influenced the submitted work.

\section{References}

1. Pappas G, Akritidis N, Bosilkovski M, Tsianos E: Brucellosis. N Engl J Med. 2005, 352:2325-2336. 10.1056/NEJMra050570

2. Brucellosis reference guide 2017. (2017). Accessed: April 20, 2020: https://www.cdc.gov/brucellosis/pdf/brucellosi-reference-guide.pdf.

3. Bosilkovski M, Dimzova M, Grozdanovski K: Natural history of brucellosis in an endemic region in different time periods. Acta Clin Croat. 2009, 48:41-46.

4. Seleem MN, Boyle SM, Sriranganathan N: Brucellosis: a re-emerging zoonosis. Vet Microbiol. 2010, 140:392 398. 10.1016/j.vetmic.2009.06.021

5. White PJ, Treanor JJ, Geremia C, Wallen RL, Blanton DW, Hallac DE: Bovine brucellosis in wildlife: using adaptive management to improve understanding, technology and suppression. Rev Sci Tech. 2013, 32:263270. 10.20506/rst.32.1.2196

6. Brucellosis in humans and animals. (2006). Accessed: April 20, 2020: https://www.who.int/csr/resources/publications/Brucellosis.pdf.

7. Sharda DC, Lubani M: A study of brucellosis in childhood . Clin Pediatr. 1986, 25:492-495. 10.1177/000992288602501002

8. Pappas G, Papadimitriou P, Akritidis N, Christou L, Tsianos EV: The new global map of human brucellosis . Lancet Infect Dis. 2006, 6:91-99. 10.1016/S1473-3099(06)70382-6

9. Mili N, Auckenthaler R, Nicod LP: Chronic brucella empyema. Chest. 1993, 103:620-621. 10.1378/chest.103.2.620

10. Spink WW: What is chronic brucellosis?. Ann Intern Med. 1951, 35:358-374. 


\section{Cureus}

11. Neglected zoonotic diseases. (2019). Accessed: April 20, 2020:

http://www.who.int/neglected_diseases/zoonoses/infections_more/en/.

12. Lim ML, Rickman LS: Brucellosis. Infect Dis Clin Pract. 2004, 12:7-14. 10.1097/01.idc.0000104894.16995.c4

13. Akhvlediani T, Clark DV, Chubabria G, Zenaishvili O, Hepburn MJ: The changing pattern of human brucellosis: clinical manifestations, epidemiology, and treatment outcomes over three decades in Georgia. BMC Infect Dis. 2010, 10:346. 10.1186/1471-2334-10-346

14. Massoud M, Kerbage F, Antoun L, Merhi R, Hallit S, Hallit R: Brucellosis: a lymphoma-like presentation. Asian Pac J Trop Dis. 2017, 10:833-834. 10.1016/j.apjtm.2017.07.025

15. Abdi-Liae Z, Soudbakhsh A, Jafari S, Tomaj K, Emadi H: Haematological manifestations of brucellosis. Acta Med Iran. 2007, 45:145-148.

16. Fanni F, Shahbaznejad L, Pourakbari B, Mahmoudi S, Mamishi S: Clinical manifestations, laboratory findings, and therapeutic regimen in hospitalized children with brucellosis in an Iranian Referral Children Medical Centre. J Health Popul Nutr. 2013, 31:218-222. 10.3329/jhpn.v31i2.16386

17. Uluğ M, Can-Uluğ N: Pulmonary involvement in brucellosis. Can J Infect Dis Med Microbiol. 2012, 23:13-15. $10.1155 / 2012 / 164892$

18. Alavi SM, Alavi L: Treatment of brucellosis: a systematic review of studies in recent twenty years . Caspian J Intern Med. 2013, 4:636-641.

19. Lytras T, Danis K, Dounias G: Incidence patterns and occupational risk factors of human brucellosis in Greece, 2004-2015. Int J Occup Environ Med. 2016, 7:221-226. 10.15171/ijoem.2016.806

20. Traxler RM, Lehman MW, Bosserman EA, Guerra MA, Smith TL: A literature review of laboratory-acquired brucellosis. J Clin Microbiol. 2013, 51:3055-3062. 10.1128/JCM.00135-13 